\title{
Rare Example of Nucleophilic Substitution at Vinylic Carbon with Inversion: Mechanism of Methyleneaziridine Formation by Sodium Amide Induced Ring Closure Revisited
}

\author{
Jason J. Shiers, Michael Shipman,* Jerome F. Hayes, and Alexandra M.Z. Slawin
}

\section{Supporting Information}

General Method A: Preparation of Allylic Amines 2, 10. To the alcohol and triethylamine (2.5 eq.) in THF ( $n \mathrm{~mL}$ ) cooled to $-30{ }^{\circ} \mathrm{C}$ was added methanesulfonyl chloride (1.05 eq.) dropwise. The reaction mixture was warmed to $0{ }^{\circ} \mathrm{C}$ over the course of $1 \mathrm{~h}$ and then maintained at this temperature for a further $30 \mathrm{~min}$. The amine (2 eq.) was added dropwise to the mixture and after stirring at $0{ }^{\circ} \mathrm{C}$ for $10 \mathrm{~min}$, the mixture heated under reflux for $18 \mathrm{~h}$. The mixture was cooled and partitioned between diethyl ether $(n \mathrm{~mL})$ and $10 \% \mathrm{NaOH}$ solution $(2 n \mathrm{~mL})$. The organic phase was washed $10 \% \mathrm{NaOH}$ solution $(2 n \mathrm{~mL})$ and brine $(2 n \mathrm{~mL})$, dried $\left(\mathrm{MgSO}_{4}\right)$ and evaporated to give the crude product which was purified by column chromatography on silica gel.

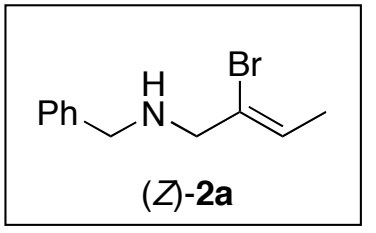

(Z)-Benzyl-(2-bromobut-2-enyl)-amine 2a. Methanesulfonyl chloride (2.40 mL, $31.0 \mathrm{mmol})$, (Z)-2-bromobut-2-en-1-ol (4.46 g, $29.5 \mathrm{mmol})$, benzylamine $(6.45 \mathrm{~mL}, 59.1 \mathrm{mmol})$ and triethylamine $(10.3 \mathrm{~mL}, 73.8$ $\mathrm{mmol})$ were reacted together in THF $(50 \mathrm{~mL})$ as described in General Method A. Purification (10\% EtOAc in petroleum ether) gave (Z)-2a (6.48 g, 92\%) as a clear colourless oil. $\mathrm{R}_{\mathrm{f}} 0.45$ (40\% EtOAc in petroleum ether); $v_{\max }$ (film) 3318, 1658, 1495, 1454 $\mathrm{cm}^{-1} ; \delta_{\mathrm{H}}\left(400 \mathrm{MHz}, \mathrm{CDCl}_{3}\right)$ 7.35-7.26 (5H, m, Ph), $5.93(1 \mathrm{H}, \mathrm{qt}, J=6.5,0.9 \mathrm{~Hz}, \mathrm{CH}), 3.71(2 \mathrm{H}$, s, $\left.\mathrm{CH}_{2} \mathrm{Ph}\right), 3.49\left(2 \mathrm{H}, \mathrm{s}, \mathrm{CH}_{2} \mathrm{CBr}\right), 2.02(1 \mathrm{H}, \mathrm{bs}, \mathrm{NH}), 1.80\left(3 \mathrm{H}, \mathrm{d}, J=6.5 \mathrm{~Hz}, \mathrm{CH}_{3}\right) ; \delta_{\mathrm{C}}(100$ $\left.\mathrm{MHz}, \mathrm{CDCl}_{3}\right) 139.8(\mathrm{C}$, aryl), $128.4(\mathrm{CH}$, aryl), $128.3(\mathrm{CH}$, aryl), $128.2(=\mathrm{CBr}), 127.1(\mathrm{CH})$, $125.7(=\mathrm{CH}), 57.1\left(\mathrm{CH}_{2} \mathrm{Ph}\right), 51.4\left(\mathrm{CBrCH}_{2}\right), 16.7\left(\mathrm{CH}_{3}\right)$; HRMS $\left(\mathrm{CI}^{+}\right)$calcd for $\mathrm{C}_{11} \mathrm{H}_{15}{ }^{79} \mathrm{BrN}$ $[\mathrm{MH}]^{+} 240.0364$, found 240.0388 .

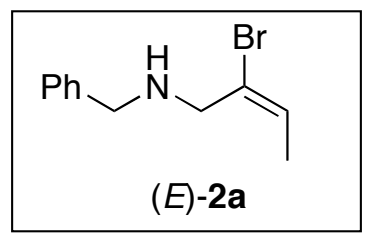

(E)-Benzyl-(2-bromobut-2-enyl)-amine 2a. Methanesulfonyl chloride (3.77 mL, $48.7 \mathrm{mmol}),(E)$-2-bromobut-2-en-1-ol (7.00 g, $46.4 \mathrm{mmol})$, benzylamine $(10.2 \mathrm{~mL}, 93.4 \mathrm{mmol})$ and triethylamine $(16.0 \mathrm{~mL}, 115$ mmol) were reacted together in THF $(100 \mathrm{~mL})$ as described in General Method A. Purification (10\% ether in petroleum ether) gave $(E)-\mathbf{2 a}(9.28 \mathrm{~g}, 83 \%)$ as a clear colourless oil. $\mathrm{R}_{\mathrm{f}} 0.20$ (40\% ether in petroleum ether); $v_{\max }$ (film) $3329,3059,3026,1644,1603$, 
$1494,1452 \mathrm{~cm}^{-1} ; \delta_{\mathrm{H}}\left(400 \mathrm{MHz}, \mathrm{CDCl}_{3}\right) 7.35-7.15(5 \mathrm{H}, \mathrm{m}, \mathrm{Ph}), 6.12(1 \mathrm{H}, \mathrm{q}, J=7.3 \mathrm{~Hz},=\mathrm{CH})$, $3.72\left(2 \mathrm{H}, \mathrm{s}, \mathrm{CH}_{2} \mathrm{Ph}\right), 3.51\left(2 \mathrm{H}, \mathrm{CH}_{2} \mathrm{CBr}\right), 2.03(1 \mathrm{H}, \mathrm{bs}, \mathrm{NH}), 1.60\left(3 \mathrm{H}, \mathrm{d}, J=7.3 \mathrm{~Hz}, \mathrm{CH}_{3}\right) ; \delta_{\mathrm{C}}$ $\left(100 \mathrm{MHz}, \mathrm{CDCl}_{3}\right) 139.7(\mathrm{C}$, aryl), $129.7(\mathrm{CH}), 128.44(\mathrm{CH}$, aryl), $128.36(\mathrm{CH}$, aryl $), 127.1$ $(=\mathrm{CH}), 125.3(=\mathrm{CBr}), 51.3\left(\mathrm{CH}_{2} \mathrm{Ph}\right), 50.1\left(\mathrm{CBrCH}_{2}\right), 15.1\left(\mathrm{CH}_{3}\right) ; \mathrm{HRMS}\left(\mathrm{EI}^{+}\right)$calcd for $\mathrm{C}_{11} \mathrm{H}_{13}{ }^{79} \mathrm{BrN}[\mathrm{M}-\mathrm{H}]^{+}$238.0231, found 238.0231. Anal. Calcd for $\mathrm{C}_{11} \mathrm{H}_{14} \mathrm{BrN}$ : C, 55.0\%; $\mathrm{H}$, 5.9\%; N, 5.8\%. Found: C, 54.7\%; H, 5.8\%; N, 5.7\%.

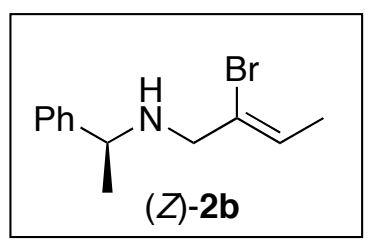

$[(Z)-2$-bromobut-2-enyl $]-[(S)$ - 1 - p h e ny let hyl $]$ - a min e 2 b . Methanesulfonyl chloride (3.07 mL, $39.7 \mathrm{mmol}),(Z)$-2-bromobut-2-en-1ol (5.70 g, $37.7 \mathrm{mmol}),(S)-1$-phenylethylamine $(9.73 \mathrm{~mL}, 75.5 \mathrm{mmol})$ and triethylamine $(13.2 \mathrm{~mL}, 94.7 \mathrm{mmol})$ were reacted together in $\mathrm{THF}$ $(75 \mathrm{~mL})$ as described in General Method A. Purification $(10 \%$ EtOAc in petroleum ether) gave $(Z)-\mathbf{2 b}(6.55 \mathrm{~g}, 68 \%)$ as a clear colourless oil. $\mathrm{R}_{\mathrm{f}} 0.46(20 \%$ EtOAc in petroleum ether); $[\alpha]_{\mathrm{D}}^{21}-55$ (c 1.0, $\left.\mathrm{CHCl}_{3}\right) ; v_{\max }$ (film) 3335, 3082, 3062, 3024, 1657, 1602, $1492,1450 \mathrm{~cm}^{-1} ; \delta_{\mathrm{H}}\left(400 \mathrm{MHz}, \mathrm{CDCl}_{3}\right) 7.36-7.23(5 \mathrm{H}, \mathrm{m}, \mathrm{Ph}), 5.79(1 \mathrm{H}, \mathrm{q}, J=6.5 \mathrm{~Hz},=\mathrm{CH})$, $3.78\left(1 \mathrm{H}, \mathrm{q}, J=6.6 \mathrm{~Hz}, \mathrm{NCHCH}_{3}\right), 3.39(1 \mathrm{H}, \mathrm{d}, J=14.5 \mathrm{~Hz}, \mathrm{NCHH}), 3.29(1 \mathrm{H}, \mathrm{d}, J=14.5 \mathrm{~Hz}$, $\mathrm{NCH} H), 1.86\left(1 \mathrm{H}\right.$, br s, NH), $1.77\left(3 \mathrm{H}, \mathrm{d}, J=6.5 \mathrm{~Hz},=\mathrm{CHCH}_{3}\right), 1.36(3 \mathrm{H}, \mathrm{d}, J=6.6 \mathrm{~Hz}$, $\left.\mathrm{CHCH}_{3}\right) ; \delta_{\mathrm{C}}\left(100 \mathrm{MHz}, \mathrm{CDCl}_{3}\right) 145.0(\mathrm{C}$, aryl $), 128.5(\mathrm{CH}$, aryl $), 128.4(=\mathrm{CBr}), 127.1(\mathrm{CH}$, aryl), $126.9(\mathrm{CH}), 125.5(\mathrm{CH}), 55.6(\mathrm{NCHAr}), 55.5\left(\mathrm{CH}_{2}\right), 24.3\left(\mathrm{NCHCH}_{3}\right), 16.6\left(=\mathrm{CHCH}_{3}\right)$; $\operatorname{MS}\left(\mathrm{CI}^{+}\right) \mathrm{m} / z 254\left(100 \%,[\mathrm{MH}]^{+}\right), 238\left(25 \%,\left[\mathrm{M}-\mathrm{CH}_{3}\right]^{+}\right), 174\left(10 \%,[\mathrm{M}-\mathrm{Br}]^{+}\right)$; HRMS $\left(\mathrm{CI}^{+}\right)$ calcd for $\mathrm{C}_{12} \mathrm{H}_{17}{ }^{79} \mathrm{BrN}[\mathrm{MH}]^{+}$254.0544, found 254.0522. Anal. Calcd for $\mathrm{C}_{12} \mathrm{H}_{16} \mathrm{BrN}$ : C, 56.7\%; H, 6.3\%; N, 5.5\%. Found: C, 56.7\%; H, 6.7\%; N, 5.5\%.

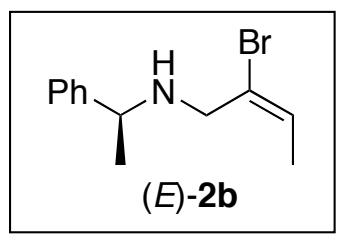

$[(E)$-2-bromobut-2-enyl $]-[(S)$ - 1 - p h e n y let hy l] - a m i n e 2 b . Methanesulfonyl chloride (1.24 mL, $16.0 \mathrm{mmol}),(E)$-2-bromobut-2-en-1-ol (2.30 g, $15.2 \mathrm{mmol}),(S)-1$-phenylethylamine $(3.93 \mathrm{~mL}, 30.5 \mathrm{mmol})$ and triethylamine $(5.30 \mathrm{~mL}, 38.0 \mathrm{mmol})$ were reacted together in THF $(30 \mathrm{~mL})$ as described in General Method A. Purification (10\% ether in petroleum ether) gave $(E)-\mathbf{2 b}(2.89 \mathrm{~g}, 75 \%)$ as a clear colourless oil. $\mathrm{R}_{\mathrm{f}} 0.32(20 \%$ EtOAc in petroleum ether); $[\alpha]_{\mathrm{D}}^{21}-36.5$ (c 1.0, $\left.\mathrm{CHCl}_{3}\right) ; v_{\max }$ (film) 3334, 3062, 3025, 1645, 1603, 1492, $1449 \mathrm{~cm}^{-1}$; $\delta_{\mathrm{H}}\left(400 \mathrm{MHz} \mathrm{CDCl}_{3}\right)$ 7.37-7.24 (5H, m, Ph), $6.05(1 \mathrm{H}, \mathrm{q}, J=7.2 \mathrm{~Hz},=\mathrm{CH}), 3.78(1 \mathrm{H}, \mathrm{q}, J=6.5$ $\left.\mathrm{Hz}, \mathrm{NCHCH}_{3}\right), 3.40(1 \mathrm{H}, \mathrm{d}, J=14.7 \mathrm{~Hz}, \mathrm{NCHH}), 3.28(1 \mathrm{H}, \mathrm{d}, J=14.7 \mathrm{~Hz}, \mathrm{NCH}), 1.92(1 \mathrm{H}$, br s, NH), $1.41\left(3 \mathrm{H}, \mathrm{d}, J=7.2 \mathrm{~Hz},=\mathrm{CHCH}_{3}\right), 1.35\left(3 \mathrm{H}, \mathrm{d}, J=6.5 \mathrm{~Hz}, \mathrm{NCHCH}_{3}\right) ; \delta_{\mathrm{C}}(100 \mathrm{MHz}$, $\left.\mathrm{CDCl}_{3}\right) 145.0(\mathrm{C}$, aryl), $129.3(\mathrm{CH}), 128.4(\mathrm{CH}$, aryl $), 127.1(\mathrm{CH}$, aryl $), 127.0(\mathrm{CH}$, aryl $), 125.8$ (C), 55.5 (NCHAr), $48.6\left(\mathrm{CH}_{2}\right), 24.8\left(\mathrm{NCHCH}_{3}\right), 14.9\left(=\mathrm{CHCH}_{3}\right) ; \mathrm{MS}\left(\mathrm{CI}^{+}\right) \mathrm{m} / z 254(100 \%$, $\left.[\mathrm{MH}]^{+}\right), 238\left(30 \%,\left[\mathrm{M}-\mathrm{CH}_{3}\right]^{+}\right)$; HRMS $\left(\mathrm{CI}^{+}\right)$calcd for $\mathrm{C}_{12} \mathrm{H}_{17}{ }^{79} \mathrm{BrN}[\mathrm{MH}]^{+} 254.0544$, found 
254.0522. Anal. Calcd for $\mathrm{C}_{12} \mathrm{H}_{16} \mathrm{BrN}$ : C, 56.7\%; H, 6.3\%; N, 5.5\%. Found: $\mathrm{C}, 56.7 \%$; $\mathrm{H}$, $6.4 \% ; \mathrm{N}, 5.2 \%$.

General Method B: Synthesis of Methyleneaziridines 1. A three-necked flask was fitted with a cold-finger condenser and a gas inlet. Iron(III) nitrate nonahydrate $(0.25 \mathrm{~mol} \%)$ was added and the system was flushed with $\mathrm{CaCl}_{2}$ dried ammonia. A dry-ice/acetone mixture was added to the condenser and ammonia (50 mL) was condensed into the flask. Freshly cut sodium (2.5 eq.) was added in small portions. The initially formed blue solution slowly faded to give a grey suspension of sodium amide. After cooling to $-78^{\circ} \mathrm{C}$, the allylamine $(50 \% \mathrm{w} / \mathrm{v}$ in diethyl ether) was added slowly to the mixture, which was subsequently stirred for $1 \mathrm{~h}$. The mixture was diluted with diethyl ether $(10 \mathrm{~mL})$ and quenched by the dropwise addition of water $(5 \mathrm{~mL})$ (CAUTION). After the ammonia had evaporated, diethyl ether $(50 \mathrm{~mL})$ was added and the mixture was stirred for $2 \mathrm{~min}$. The organic phase was separated and washed $0.1 \mathrm{M}$ acetic acid (5 x $50 \mathrm{~mL})$, then $10 \% \mathrm{NaOH}(2 \times 50 \mathrm{~mL})$ and finally brine $(50 \mathrm{~mL})$, then dried $\left(\mathrm{MgSO}_{4}\right)$. Removal of the solvent under reduced pressure and subsequent bulb-to-bulb distillation yielded the product.

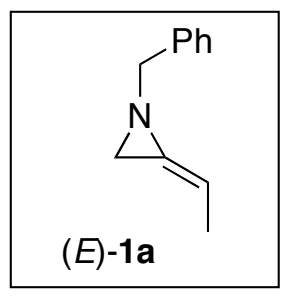

(E)-1-Benzyl-2-ethylideneaziridine 1a. Generation of sodium amide from sodium (600 mg, $26.1 \mathrm{mmol})$ and iron(III) nitrate nonahydrate $(12.0 \mathrm{mg}, 0.03$ $\mathrm{mmol})$ in ammonia $(50 \mathrm{~mL})$ and subsequent reaction with $(Z)-2 \mathrm{a}(2.50 \mathrm{~g}, 10.4$ mmol) for $1 \mathrm{~h}$ at $-78^{\circ} \mathrm{C}$ as described in General Method $\mathrm{B}$, followed by workup and bulb-to-bulb distillation $\left(\mathrm{ca} 60{ }^{\circ} \mathrm{C} / 1 \mathrm{mmHg}\right)$ gave $(E)-\mathbf{1 a}(603 \mathrm{mg}$, $37 \%)$ as a pale yellow oil. $\mathrm{R}_{\mathrm{f}} 0.54\left(40 \%\right.$ EtOAc in petroleum ether); $v_{\max }$ (film) $1781,1496,1453 \mathrm{~cm}^{-1} ; \delta_{\mathrm{H}}\left(400 \mathrm{MHz}, \mathrm{CDCl}_{3}\right) 7.45-7.25(5 \mathrm{H}, \mathrm{m}, \mathrm{Ph}), 5.20(1 \mathrm{H}, \mathrm{qt}, J=6.7$, $1.4 \mathrm{~Hz},=\mathrm{CH}), 3.64\left(2 \mathrm{H}\right.$, br s, $\left.\mathrm{NCH}_{2} \mathrm{Ph}\right), 2.18(2 \mathrm{H}$, br s, aziridine $), 1.78(3 \mathrm{H}, \mathrm{d}, J=6.7 \mathrm{~Hz}$, $\left.=\mathrm{CHCH}_{3}\right) ; \delta_{\mathrm{C}}\left(75 \mathrm{MHz}, \mathrm{CDCl}_{3}\right) 138.7(\mathrm{C}$, aryl), $130.1(=\mathrm{CN}), 128.4(\mathrm{CH}$, aryl $), 128.2(\mathrm{CH}$, aryl), $127.2(\mathrm{CH}$, aryl $), 95.0(=\mathrm{CH}), 63.4\left(\mathrm{CH}_{2} \mathrm{Ph}\right), 30.5\left(\mathrm{CH}_{2}\right.$, aziridine $), 14.2\left(\mathrm{CH}_{3}\right)$; HRMS $\left(\mathrm{CI}^{+}\right)$calcd for $\mathrm{C}_{11} \mathrm{H}_{14} \mathrm{~N}[\mathrm{MH}]^{+} 160.1126$, found 160.1119 .

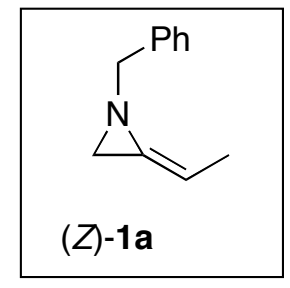

(Z)-1-Benzyl-2-ethylideneaziridine 1a. Generation of sodium amide from sodium (600 mg, $26.1 \mathrm{mmol})$ and iron(III) nitrate nonahydrate $(12.0 \mathrm{mg}$, $0.03 \mathrm{mmol})$ in ammonia $(50 \mathrm{~mL})$ and subsequent reaction with $(E)-\mathbf{2 a}(2.50$ $\mathrm{g}, 10.4 \mathrm{mmol}$ ) for $1 \mathrm{~h}$ at $-78^{\circ} \mathrm{C}$ in ammonia as described in General Method $\mathrm{B}$, followed by work-up and bulb-to-bulb distillation ( $\mathrm{ca} 50{ }^{\circ} \mathrm{C} / 0.4 \mathrm{mmHg}$ ) gave $(Z)-\mathbf{1 a}(1.28 \mathrm{~g}, 77 \%)$ as a pale yellow oil. The isolated $(Z)-\mathbf{1 a}$ contained $(E)-\mathbf{1 a}(8 \%)$ which 
reflects the presence of $(Z)-\mathbf{2 a}(7 \%)$ in the starting material. $\mathrm{R}_{\mathrm{f}} 0.42(40 \%$ EtOAc in petroleum ether); $v_{\max }$ (film) 1778, 1495, $1453 \mathrm{~cm}^{-1} ; \delta_{\mathrm{H}}\left(400 \mathrm{MHz}, \mathrm{CDCl}_{3}\right)$ 7.39-7.24 (5H, m, Ph), 5.11 $\left(1 \mathrm{H}, \mathrm{q}, J=6.8 \mathrm{~Hz},=\mathrm{CHCH}_{3}\right), 3.71\left(2 \mathrm{H}, \mathrm{br} \mathrm{s}, \mathrm{CH}_{2} \mathrm{Ph}\right), 2.10(2 \mathrm{H}$, br s, aziridine $), 1.70(3 \mathrm{H}, \mathrm{d}, J=$ $\left.6.8 \mathrm{~Hz}, \mathrm{CH}_{3}\right) ; \delta_{\mathrm{C}}\left(75 \mathrm{MHz}, \mathrm{CDCl}_{3}\right) 138.5(\mathrm{C}$, aryl), $129.9(=\mathrm{CN}), 128.4(\mathrm{CH}$, aryl $), 128.3(\mathrm{CH}$, aryl), $127.3(\mathrm{CH}$, aryl $), 95.2\left(=\mathrm{CHCH}_{3}\right), 62.1\left(\mathrm{CH}_{2} \mathrm{Ph}\right), 31.3\left(\mathrm{CH}_{2}\right.$, aziridine $), 13.4\left(\mathrm{CH}_{3}\right) ; \mathrm{MS}$ $\left(\mathrm{EI}^{+}\right) 159\left(6 \%, \mathrm{M}^{+}\right), 91\left(100 \%,\left[\mathrm{PhCH}_{2}\right]^{+}\right)$; HRMS $\left(\mathrm{CI}^{+}\right)$calcd for $\mathrm{C}_{11} \mathrm{H}_{14} \mathrm{~N}[\mathrm{MH}]^{+} 160.1121$, found 160.1120 .

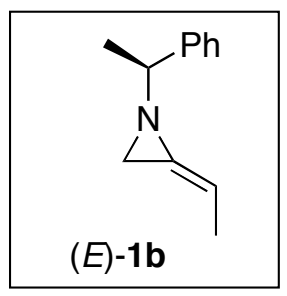

(E)-1-[(S)-1-phenylethyl]-2-ethylideneaziridine 1b. Generation of sodium amide from sodium (500 mg, $21.7 \mathrm{mmol}$ ) and iron(III) nitrate nonahydrate $(8.8 \mathrm{mg}, 0.02 \mathrm{mmol})$ in ammonia $(50 \mathrm{~mL})$ and subsequent reaction with $(Z)-\mathbf{2 b}$ (2.21 g, $8.69 \mathrm{mmol})$ for $1 \mathrm{~h}$ at $-78{ }^{\circ} \mathrm{C}$ as described in General Method B, followed by work-up and bulb-to-bulb distillation $\left(\mathrm{ca} 50^{\circ} \mathrm{C} / 0.1 \mathrm{mmHg}\right)$ gave $(E)-\mathbf{1 b}(865 \mathrm{mg}, 57 \%)$ as a pale yellow oil. The isolated $(E)-\mathbf{1 b}$ contained $(Z)$ $1 \mathbf{b}(6 \%)$ which reflects the presence of $(E)-\mathbf{2 b}(6 \%)$ in the starting material. $\mathrm{R}_{\mathrm{f}} 0.46(20 \%$ EtOAc in petroleum ether); $[\alpha]_{\mathrm{D}}^{21}-152\left(c 1.0, \mathrm{CHCl}_{3}\right) ; v_{\max }\left(\right.$ film) $1777,1604,1492,1450 \mathrm{~cm}^{-1}$; $\delta_{\mathrm{H}}\left(400 \mathrm{MHz}, \mathrm{d}_{6}\right.$-DMSO) 7.36-7.21 (5H, m, Ph), $4.98(1 \mathrm{H}, \mathrm{br} \mathrm{s},=\mathrm{CH}), 2.84(1 \mathrm{H}, \mathrm{q}, J=6.6 \mathrm{~Hz}$, $\left.\mathrm{NCHCH}_{3}\right), 2.05(1 \mathrm{H}$, br s, aziridine), $1.95(1 \mathrm{H}$, br s, aziridine $), 1.66(3 \mathrm{H}, \mathrm{d}, J=6.6 \mathrm{~Hz}$, $\left.=\mathrm{CHCH}_{3}\right), 1.36\left(3 \mathrm{H}, \mathrm{d}, J=6.6 \mathrm{~Hz}, \mathrm{CHCH}_{3} \mathrm{Ph}\right) ; \delta_{\mathrm{C}}\left(100 \mathrm{MHz}, \mathrm{d}_{6}-\mathrm{DMSO}\right) 144.7$ (C, aryl), 131.3 $(=\mathrm{CN}), 128.6(\mathrm{CH}$, aryl $), 127.4(\mathrm{CH}$, aryl $), 127.1(\mathrm{CH}$, aryl $), 93.1\left(=\mathrm{CHCH}_{3}\right), 68.1(\mathrm{CHAr}), 29.3$ $\left(\mathrm{CH}_{2}\right.$, ring), $23.8\left(\mathrm{CHCH}_{3} \mathrm{Ph}\right), 14.7\left(=\mathrm{CHCH}_{3}\right)$; $\mathrm{HRMS}\left(\mathrm{CI}^{+}\right)$calcd for $\mathrm{C}_{12} \mathrm{H}_{16} \mathrm{~N}[\mathrm{MH}]^{+} 174.1283$, found 174.1278. Enantiomeric excess determined by treatment of $(E)-\mathbf{1 b}(5 \mathrm{mg})$ with $(S)-2,2,2-$ trifluoro-1-(9-anthryl)-ethanol $(16 \mathrm{mg})$ in $\mathrm{CDCl}_{3}(0.75 \mathrm{~mL})$ and subsequent integration of the following ${ }^{1} \mathrm{H}$ NMR regions: $\delta_{\mathrm{H}}(500 \mathrm{MHz}) 1.375-1.345(0.03 \mathrm{H}$, minor), 1.335-1.295 $(0.97 \mathrm{H}$, major). Racemic material used to spike the mixture and verify the analysis.

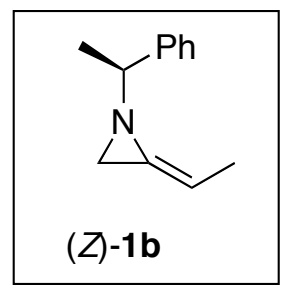

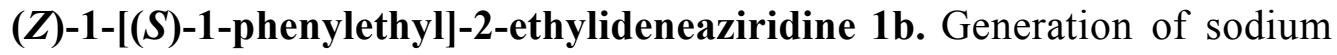
amide from sodium $(600 \mathrm{mg}, 26.1 \mathrm{mmol})$ and iron(III) nitrate nonahydrate $(12.0 \mathrm{mg}, 0.03 \mathrm{mmol})$ in ammonia $(50 \mathrm{~mL})$ and subsequent reaction with $(E)$ 2b (2.64 $\mathrm{g}, 10.4 \mathrm{mmol})$ for $1 \mathrm{~h}$ at $-78^{\circ} \mathrm{C}$ as described in General Method B, followed by work-up and bulb-to-bulb distillation $\left(\mathrm{ca} 75^{\circ} \mathrm{C} / 1 \mathrm{mmHg}\right)$ gave $(Z)-\mathbf{1 b}(1.67 \mathrm{~g}, 93 \%)$ as a pale yellow oil. The isolated $(Z)-\mathbf{1 b}$ contained $(E)-\mathbf{1 b}$ $(9 \%)$ which reflects the presence of $(Z)-\mathbf{2 b}(8 \%)$ in the starting material. $\mathrm{R}_{\mathrm{f}} 0.46(20 \%$ EtOAc in petroleum ether); $[\alpha]_{\mathrm{D}}^{21}-213\left(c 1.0, \mathrm{CHCl}_{3}\right) ; v_{\max }\left(\right.$ film) $1780,1601,1493,1450 \mathrm{~cm}^{-1} ; \delta_{\mathrm{H}}(400$ MHz, d -DMSO$_{6}$ 7.38-7.22 (5H, m, Ph), $4.93(1 \mathrm{H}, \mathrm{q}, J=6.7 \mathrm{~Hz},=\mathrm{CH}), 2.98(1 \mathrm{H}, \mathrm{q}, J=6.6 \mathrm{~Hz}$, $\left.\mathrm{NCHCH}_{3}\right), 2.06(1 \mathrm{H}$, br s, aziridine), $1.94(1 \mathrm{H}$, br s, aziridine $), 1.39(3 \mathrm{H}, \mathrm{d}, J=6.6 \mathrm{~Hz}$, 
$\left.\mathrm{CHCH}_{3} \mathrm{Ph}\right), 1.26\left(3 \mathrm{H}\right.$, br s, $\left.=\mathrm{CHCH}_{3}\right) ; \delta_{\mathrm{C}}\left(100 \mathrm{MHz}, \mathrm{d}_{6}-\mathrm{DMSO}\right) 145.0(\mathrm{C}$, aryl $), 130.6(=\mathrm{CN})$, $128.7(\mathrm{CH}$, aryl $), 127.5\left(\mathrm{CH}\right.$, aryl), $127.4\left(\mathrm{CH}\right.$, aryl), $93.9\left(=\mathrm{CHCH}_{3}\right), 67.4(\mathrm{CHAr}), 29.4\left(\mathrm{CH}_{2}\right.$, aziridine), $23.9\left(\mathrm{CHCH}_{3} \mathrm{Ph}\right), 14.2\left(=\mathrm{CHCH}_{3}\right)$; $\mathrm{HRMS}\left(\mathrm{CI}^{+}\right)$calcd for $\mathrm{C}_{12} \mathrm{H}_{16} \mathrm{~N}[\mathrm{MH}]^{+} 174.1283$, found 174.1290. Enantiomeric excess determined by treatment of $(Z)-\mathbf{1 b}(5 \mathrm{mg})$ with $(S)$-2,2,2trifluoro-1-(9-anthryl)-ethanol $(32 \mathrm{mg})$ in $\mathrm{CDCl}_{3}(0.75 \mathrm{~mL})$ and subsequent integration of the

following ${ }^{1} \mathrm{H}$ NMR regions: $\delta_{\mathrm{H}}(500 \mathrm{MHz}) 1.485-1.450(0.05 \mathrm{H}$, minor), $1.450-1.415(0.95 \mathrm{H}$, major). Racemic material used to spike the mixture and verify the analysis.

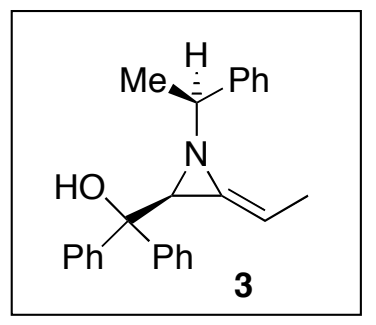

[(Z)-2-ethylidene-1-[(S)-1-phenylethyl]aziridin-3-

yl]diphenylmethanol 3. To a stirred solution of (Z)-1b (218 mg, 1.26 mmol) and TMEDA $(227 \mu 1,1.50 \mathrm{mmol})$ in THF $(10 \mathrm{~mL})$ at $-78{ }^{\circ} \mathrm{C}$ was added sec-butyllithium (1.3M in hexanes, $1.25 \mathrm{~mL}, 1.63 \mathrm{mmol})$ dropwise. The resulting solution was stirred at $-78{ }^{\circ} \mathrm{C}$ for $5 \mathrm{~h}$, then benzophenone (297 mg, $1.63 \mathrm{mmol})$ in THF (1 mL) added dropwise. After $10 \mathrm{~min}$, the mixture was warmed to room temperature and stirred overnight. The mixture was partitioned between water $(25 \mathrm{~mL})$ and diethyl ether $(25 \mathrm{~mL})$, and the aqueous layer washed with diethyl ether $(2 \times 25 \mathrm{~mL})$. The combined organic phases were washed with brine $(75 \mathrm{~mL})$, dried $\left(\mathrm{MgSO}_{4}\right)$ and the solvent removed in vacuo. To remove unreacted benzophenone, the crude product was dissolved in ethanol $(20 \mathrm{~mL})$ and cooled to $0{ }^{\circ} \mathrm{C}$. Sodium borohydride (108 $\mathrm{mg}, 2.85 \mathrm{mmol}$ ) was added and the mixture warmed to room temperature and stirred for $4 \mathrm{~h}$. The reaction was quenched by the dropwise addition of ammonium chloride solution, then basified using sodium hydrogen carbonate solution. The mixture was extracted with diethyl ether $(3 \times 25 \mathrm{~mL})$ and the combined organic extracts washed with brine $(75 \mathrm{~mL})$, dried $\left(\mathrm{MgSO}_{4}\right)$ and the solvent removed in vacuo. Column chromatography (10\% ether in petroleum ether) gave 3 (371 mg, 83\%) as a colourless oil. Crystals suitable for X-ray crystallography were grown from diethyl ether (slow evaporation). $\mathrm{R}_{\mathrm{f}} 0.35$ (20\% $\mathrm{Et}_{2} \mathrm{O}$ in petroleum ether); $[\alpha]_{\mathrm{D}}^{21}-11.4$ (c 2.2, $\mathrm{CHCl}_{3}$ ); m.p. $102-105^{\circ} \mathrm{C} ; v_{\max }$ (film) 3422, 3053, 3028, 1782, 1595. 1490, $1445 \mathrm{~cm}^{-1} ; \delta_{\mathrm{H}}(300$ $\left.\mathrm{MHz}_{\mathrm{CDCl}}\right)$ 7.55-7.20 (15H, m, Ph), $5.09\left(1 \mathrm{H}, \mathrm{q}, J=6.6 \mathrm{~Hz},=\mathrm{CHCH}_{3}\right), 3.88(1 \mathrm{H}, \mathrm{s}, \mathrm{OH}), 3.16$ $\left(1 \mathrm{H}, \mathrm{q}, J=6.8 \mathrm{~Hz}, \mathrm{CHCH}_{3} \mathrm{Ph}\right), 2.93(1 \mathrm{H}, \mathrm{s}$, aziridine $), 1.08,\left(3 \mathrm{H}, \mathrm{d}, J=6.8 \mathrm{~Hz},=\mathrm{CHCH}_{3}\right), 0.85$ $\left(3 \mathrm{H}, \mathrm{d}, J=6.6 \mathrm{~Hz}, \mathrm{NCHCH}_{3}\right) ; \delta_{\mathrm{C}}\left(75 \mathrm{MHz}^{\mathrm{CDCl}} \mathrm{CD}_{3}\right) 146.7$ (C, aryl), 144.5 (C, aryl), $143.8(\mathrm{C}$, aryl), $129.3(=\mathrm{CN}), 128.2(\mathrm{CH}$, aryl $), 128.0(\mathrm{CH}$, aryl), $127.8(\mathrm{CH}$, aryl $), 127.23(\mathrm{CH}$, aryl), $127.17(\mathrm{CH}, \operatorname{aryl}), 127.0(\mathrm{CH}$, aryl $), 126.9(\mathrm{CH}, \operatorname{aryl}), 126.7(\mathrm{CH}$, aryl $), 126.0(\mathrm{CH}$, aryl $), 97.2$ $\left(=\mathrm{CHCH}_{3}\right), 74.6\left(\mathrm{CPh}_{2} \mathrm{OH}\right), 66.3\left(\mathrm{CHCH}_{3} \mathrm{Ph}\right), 50.0$ (aziridine), $22.9\left(\mathrm{NCHCH}_{3}\right), 12.9\left(=\mathrm{CHCH}_{3}\right)$; MS $\left(\mathrm{EI}^{+}\right) \mathrm{m} / z 355\left(30 \%, \mathrm{M}^{+}\right), 250\left(70 \%,\left[\mathrm{M}-\mathrm{PhCHCH}_{3}\right]^{+}\right), 105\left(100 \%,\left[\mathrm{PhCHCH}_{3}\right]^{+}\right) ; \mathrm{HRMS}$ $\left(\mathrm{EI}^{+}\right.$) calcd for $\mathrm{C}_{25} \mathrm{H}_{25} \mathrm{NO}[\mathrm{M}]^{+}$355.1936, found 355.1918; Anal. Calcd for $\mathrm{C}_{25} \mathrm{H}_{25} \mathrm{NO}$ : C, 84.5; $\mathrm{H}$, $7.1 ; \mathrm{N}, 3.9 \%$. Found: C, 84.4; H, 7.1; N, 3.9\%. 


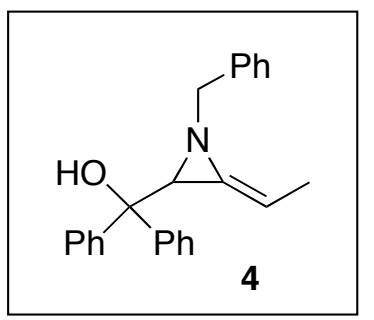

[(Z)-1-benzyl-2-ethylideneaziridin-3-yl]diphenylmethanol 4. To a stirred solution of $(Z)-\mathbf{1 a}(200 \mathrm{mg}, 1.26 \mathrm{mmol})$ and TMEDA $(227 \mu \mathrm{l}$, $1.50 \mathrm{mmol})$ in THF $(10 \mathrm{~mL})$ at $-78{ }^{\circ} \mathrm{C}$ was added sec-butyllithium $(1.3 \mathrm{M}$ in hexanes, $1.25 \mathrm{~mL}, 1.63 \mathrm{mmol})$ dropwise. The resulting solution was stirred at $-78{ }^{\circ} \mathrm{C}$ for $5 \mathrm{~h}$, then benzophenone $(297 \mathrm{mg}, 1.63 \mathrm{mmol})$ in THF $(1 \mathrm{~mL})$ added dropwise. After $10 \mathrm{~min}$, the mixture was warmed to room temperature and stirred overnight. The mixture was partitioned between water $(25 \mathrm{~mL})$ and ether $(25 \mathrm{~mL})$, and the aqueous layer washed with diethyl ether $(2 \times 25 \mathrm{~mL})$. The combined organic phases were washed with brine $(75 \mathrm{~mL})$, dried $\left(\mathrm{MgSO}_{4}\right)$ and the solvent removed in vасио. To remove unreacted benzophenone, the crude product was dissolved in ethanol $(20 \mathrm{~mL})$ and cooled to $0{ }^{\circ} \mathrm{C}$. Sodium borohydride $(108 \mathrm{mg}, 2.85 \mathrm{mmol})$ was added and the mixture warmed to room temperature and stirred for $4 \mathrm{~h}$. The reaction was quenched by the dropwise addition of ammonium chloride solution, then basified using sodium hydrogen carbonate solution. The mixture was extracted with diethyl ether $(3 \times 25 \mathrm{~mL})$ and the combined organic extracts washed with brine $(75 \mathrm{~mL})$, dried $\left(\mathrm{MgSO}_{4}\right)$ and the solvent removed in vacuo. Column chromatography (2\% EtOAc in petroleum ether) gave 4 ( $267 \mathrm{mg}, 62 \%$ ) as a colourless oil, which crystallised on standing. Crystals suitable for X-ray crystallography were grown from diethyl ether (slow evaporation). $\mathrm{R}_{\mathrm{f}} 0.46\left(40 \% \mathrm{Et}_{2} \mathrm{O}\right.$ in petroleum ether); m.p. $87-89^{\circ} \mathrm{C} ; v_{\max }$ (film) $3444,3058,3028,1785,1600.1493,1447 \mathrm{~cm}^{-1} ; \delta_{\mathrm{H}}\left(400 \mathrm{MHz}, \mathrm{CDCl}_{3}\right) 7.36-7.08(15 \mathrm{H}, \mathrm{m}, \mathrm{Ph})$, $5.13(1 \mathrm{H}, \mathrm{q}, J=6.8 \mathrm{~Hz},=\mathrm{CH}), 4.03(1 \mathrm{H}, \mathrm{d}, J=13.1 \mathrm{~Hz}, \mathrm{C} H \mathrm{HPh}), 3.66(1 \mathrm{H}, \mathrm{s}, \mathrm{OH}), 3.63(1 \mathrm{H}, \mathrm{d}$, $J=13.1 \mathrm{~Hz}, \mathrm{CH} H \mathrm{Ph}), 2.93(1 \mathrm{H}, \mathrm{s}$, aziridine $), 1.69,\left(3 \mathrm{H}, \mathrm{d}, J=6.8 \mathrm{~Hz}, \mathrm{CH}_{3}\right) ; \delta_{\mathrm{C}}(75 \mathrm{MHz}$, $\left.\mathrm{CDCl}_{3}\right) 146.4(\mathrm{C}, \operatorname{aryl}), 144.7(\mathrm{C}, \operatorname{aryl}), 137.0(\mathrm{C}, \operatorname{aryl}), 129.8(=\mathrm{CN}), 128.9(\mathrm{CH}$, aryl $), 128.3$ $(\mathrm{CH}$, aryl), $128.1(\mathrm{CH}$, aryl), $128.0(\mathrm{CH}$, aryl), $127.5(\mathrm{CH}$, aryl $), 127.3(\mathrm{CH}$, aryl $), 126.8(\mathrm{CH}$, aryl), $126.3\left(\mathrm{CH}\right.$, aryl), $97.0\left(=\mathrm{CHCH}_{3}\right), 74.8\left(\mathrm{CPh}_{2} \mathrm{OH}\right), 60.3\left(\mathrm{CH}_{2} \mathrm{Ph}\right), 50.2\left(\mathrm{CHCPh}{ }_{2} \mathrm{OH}\right), 13.4$ $\left(\mathrm{CH}_{3}\right)$; HRMS $\left(\mathrm{ES}^{+}\right)$calcd for $\mathrm{C}_{24} \mathrm{H}_{24} \mathrm{NO}[\mathrm{MH}]^{+} 342.1852$, found 342.1855; Anal. Calcd for $\mathrm{C}_{24} \mathrm{H}_{23} \mathrm{NO}$ : C, $84.4 ; \mathrm{H}, 6.8 ; \mathrm{N}, 4.1 \%$. Found: $\mathrm{C}, 84.3 ; \mathrm{H}, 6.8 ; \mathrm{N}, 4.0 \%$.

X-ray Crystallographic Structure Determinations. 3: $\mathrm{C}_{25} \mathrm{H}_{25} \mathrm{NO}$, colourless prism, $0.1 \times 0.1 \times 0.1 \mathrm{~mm}$, orthorhombic, space group $P 2_{1}{ }_{1}{ }_{1}{ }_{1}, Z=4, a=9.8665(15), b=12.1832(18), c=$ 16.897(3) $\AA, V=2031.2(5) \AA^{3}, \rho_{\text {calcd }} 1.162 \mathrm{Mgm}^{-3}, 2 \theta_{\max }=51^{\circ}$, graphite monochramated $\mathrm{Mo}_{\mathrm{K}_{\alpha}}$ radiation $(\lambda=0.71073 \AA), \mu\left(\mathrm{Mo}_{\mathrm{K}_{\alpha}}\right)=0.070 \mathrm{~mm}^{-1}, T=125 \mathrm{~K}$. Bruker SMART CCD, Lorentzian, polarization and absorption corrections applied (max./min. transmission 1.0000/0.587127). Of 12970 measured data, 3680 were unique and 3075 observed $[I>2 \sigma(I)] . R_{1}=0.0368$ and $w R_{2}=$ 0.0763 for 250 parameters with a residual electron extremes of -0.151 and 0.159 e $\AA^{-3}$ Flack 
parameter $-0.7(14)$. 4: $\mathrm{C}_{24} \mathrm{H}_{23} \mathrm{NO}$, colourless prism, $0.1 \times 0.1 \times 0.1 \mathrm{~mm}$, monoclinic, space group $P 2_{1} / c, Z=4, a=7.4715(8), b=11.4568(12), c=21.543(2) \AA, V=1838.8(3) \AA^{3}, \rho_{\text {calcd }} 1.233$ $\mathrm{Mgm}^{-3}, 2 \theta_{\max }=50.8^{\circ}$, graphite monochramated $\mathrm{Mo}_{\mathrm{K}_{\alpha}}$ radiation $(\lambda=0.71073 \AA), \mu\left(\mathrm{Mo}_{\mathrm{K}_{\alpha}}\right)$ $=0.074 \mathrm{~mm}^{-1}, T=125 \mathrm{~K}$. Bruker SMART CCD, Lorentzian, polarization and absorption corrections applied (max./min. transmission 1.0000/0.922477). Of 11001 measured data, 3304 were unique and 2932 observed $[I>2 \sigma(I)] . \quad R_{1}=0.0331$ and $w R_{2}=0.0788$ for 241 parameters with a residual electron extremes of -0.181 and $0.271 \mathrm{e}^{-3}$. The structures were solved by direct methods. The non-hydrogen atoms were refined anisotropically, the C-H hydrogen atoms were idealized and refined isotropically using a riding model. Structural refinements were performed with the full-matrix least-squares method on $F^{2}$ for all data (G M Sheldrick, SHELXTL Version 6.10, Bruker AXS, Madison WI, 2002).

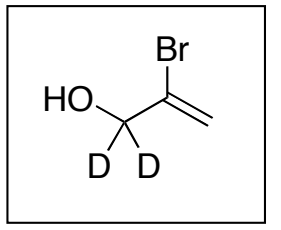

2-Bromo-1,1-dideuteroprop-2-en-1-ol. Aluminium chloride (1.73 g, 13.0 $\mathrm{mmol}$ ) was added portionwise to a vigourously stirred solution of lithium aluminium deuteride $(1.60 \mathrm{~g}, 38.1 \mathrm{mmol})$ in diethyl ether $(200 \mathrm{~mL})$ at $0{ }^{\circ} \mathrm{C}$. The resulting mixture was allowed to warm to room temperature. After stirring for $10 \mathrm{~min}$, the mixture was cooled to $-78{ }^{\circ} \mathrm{C}$ and ethyl 2-bromoacrylate $(9.12 \mathrm{~g}, 50.9 \mathrm{mmol})$ in diethyl ether $(30 \mathrm{~mL})$ was added dropwise. The mixture was warmed to $0{ }^{\circ} \mathrm{C}$, stirred for $1 \mathrm{~h}$ then quenched by dropwise addition of water $(7 \mathrm{~mL})$, followed by $10 \% \mathrm{NaOH}$ solution $(3 \mathrm{~mL})$. The mixture was warmed to room temperature whereupon it was filtered and the precipitate washed with diethyl ether $(100 \mathrm{~mL})$. The supernatant was dried $\left(\mathrm{MgSO}_{4}\right)$, filtered and evaporated. Column chromatography ( $10 \%$ ether in petroleum ether) gave the title compound (5.29 g, 75\%) as a clear colourless oil. $\mathrm{R}_{\mathrm{f}} 0.35$ (20\% diethyl ether in petroleum ether); $v_{\max }$ (film) 3286,1632 $\mathrm{cm}^{-1} ; \delta_{\mathrm{H}}\left(400 \mathrm{MHz}, \mathrm{CDCl}_{3}\right) 5.93(1 \mathrm{H}, \mathrm{d},=\mathrm{CHH}), 5.58(1 \mathrm{H}, \mathrm{s},=\mathrm{CH} H), 2.52(1 \mathrm{H}, \mathrm{br} \mathrm{s}, \mathrm{OH}) ; \delta_{\mathrm{C}}$ $\left(100 \mathrm{MHz}, \mathrm{CDCl}_{3}\right) 132.3$ (=CBr), $116.4\left(\mathrm{CH}_{2}\right), 66.8\left(\mathrm{CD}_{2}\right)$; GC-MS $\left(\mathrm{EI}^{+}\right) \mathrm{m} / z 138\left(20 \%, \mathrm{M}^{+}\right)$, $\left.59.1(100 \% \text {, [M-Br] }]^{+}\right)$; HRMS $\left(\mathrm{EI}^{+}\right)$calcd for $\mathrm{C}_{3} \mathrm{H}_{3} \mathrm{BrD}_{2} \mathrm{O}[\mathrm{M}]^{+}$137.9644, found 137.9645 .

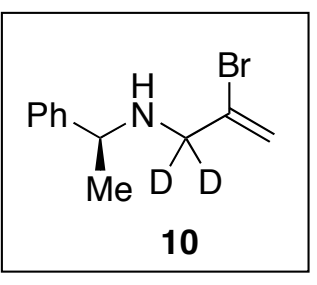

(2-Bromo-1,1-dideuteroprop-2-enyl)-[(S)-1-phenylethyl]-amine 10. Methane-sulfonyl chloride $(2.84 \mathrm{~mL}, 36.7 \mathrm{mmol})$, 2-bromo-1,1dideuteroprop-2-en-1-ol (4.75 g, $34.2 \mathrm{mmol}), \alpha$-methylbenzylamine (8.94 $\mathrm{mL}, 69.3 \mathrm{mmol})$ and triethylamine $(12.0 \mathrm{~mL}, 86.1 \mathrm{mmol})$ were reacted together in THF $(75 \mathrm{~mL})$ as described in General Method A. Purification (10\% EtOAc in petroleum ether) gave $\mathbf{1 0}(5.73 \mathrm{~g}, 69 \%)$ as a clear colourless oil. Material judged to have $95 \% \mathrm{D}$ incorporation by integration of the residual hydrogen signals at $\delta 3.36$ and 3.25 in the ${ }^{1} \mathrm{H}$ NMR spectrum. $[\alpha]_{\mathrm{D}}^{21}-31.6\left(c 10, \mathrm{CHCl}_{3}\right) ; \mathrm{R}_{\mathrm{f}} 0.41$ (10\% EtOAc in petroleum ether); $v_{\max }$ 
(film) $3334,3060,1624,1492,1450 \mathrm{~cm}^{-1} ; \delta_{\mathrm{H}}\left(300 \mathrm{MHz}, \mathrm{CDCl}_{3}\right) 7.29-7.20(5 \mathrm{H}, \mathrm{m}$, aromatics), $5.66(1 \mathrm{H}, \mathrm{d}, J=1.8 \mathrm{~Hz},=\mathrm{CHH}), 5.55(1 \mathrm{H}, \mathrm{d}, J=1.8 \mathrm{~Hz},=\mathrm{CH} H), 3.79(1 \mathrm{H}, \mathrm{q}, J=6.6 \mathrm{~Hz}$, $\left.\mathrm{CHCH}_{3} \mathrm{Ph}\right), 1.77(1 \mathrm{H}, \mathrm{bs}, \mathrm{NH}), 1.35\left(3 \mathrm{H}, \mathrm{d}, J=6.6 \mathrm{~Hz}, \mathrm{CHCH}_{3} \mathrm{Ph}\right) ; \delta_{\mathrm{C}}\left(75 \mathrm{MHz}, \mathrm{CDCl}_{3}\right) 145.2$ $\left(\mathrm{C}\right.$, aryl), 134.2 (=CBr), $128.9(\mathrm{CH}$, aryl $), 127.5(\mathrm{CH}$, aryl $), 127.3(\mathrm{CH}$, aryl $), 118.2\left(=\mathrm{CH}_{2}\right), 55.9$ $\left(\mathrm{CHCH}_{3} \mathrm{Ph}\right), 55.1\left(\mathrm{CD}_{2}\right), 24.7\left(\mathrm{CH}_{3}\right) ; \delta_{\mathrm{D}}\left(75 \mathrm{MHz}, \mathrm{CHCl}_{3}\right) 3.33$ (s), $3.23(\mathrm{~s})$; HRMS $\left(\mathrm{EI}^{+}\right)$calcd for $\mathrm{C}_{11} \mathrm{H}_{12} \mathrm{D}_{2} \mathrm{~N}^{79} \mathrm{Br}[\mathrm{M}]^{+}$241.0435, found 241.0441.

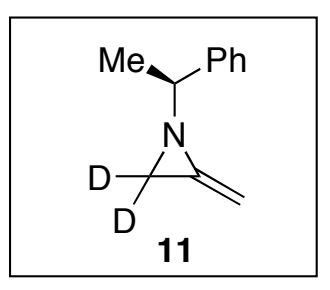

3,3-Dideutero-2-methylene-1-[(1S)-1-phenylethyl]aziridine 11. A threenecked flask was fitted with a dry-ice condenser and a gas inlet. Iron(III) chloride $\left(2.0 \mathrm{mg}, 0.01 \mathrm{mmol}\right.$ ) was added and the system purged with $\mathrm{CaCl}_{2}$ dried ammonia. A dry-ice/acetone mixture was added to the condenser and ammonia $(25 \mathrm{~mL})$ condensed into the flask. Freshly cut sodium $(118 \mathrm{mg}$, $5.13 \mathrm{mmol}$ ) was added in small portions. The initially formed blue solution slowly faded to give a grey suspension of sodium amide. $10(1.00 \mathrm{~g}, 4.13 \mathrm{mmol})$ in ether $(1 \mathrm{~mL})$ was added slowly to the mixture, which was then stirred for $5 \mathrm{~min}$. The mixture was diluted with diethyl ether $(5 \mathrm{~mL})$ and quenched by the dropwise addition of water $(2 \mathrm{~mL})$ (CAUTION). After the ammonia had evaporated, diethyl ether $(25 \mathrm{~mL})$ was added and the mixture was stirred for $2 \mathrm{~min}$. The organic phase was separated and washed with $0.1 \mathrm{M}$ acetic acid $(3 \times 50 \mathrm{~mL}), 10 \% \mathrm{NaOH}(2 \times 50 \mathrm{~mL})$ and brine $(50 \mathrm{~mL})$, then dried $\left(\mathrm{MgSO}_{4}\right)$. Removal of the solvent in vacuo and subsequent bulb-tobulb distillation gave 11 (565 mg, 85\%) as a clear colourless oil. Material judged to have $92 \% \mathrm{D}$ incorporation by integration of the residual hydrogen signals at 2.11, 2.09, 2.01 and $2.00 \mathrm{ppm}$ in the ${ }^{1} \mathrm{H}$ NMR spectrum (average of three experiments). $\mathrm{R}_{\mathrm{f}} 0.52$ (10\% EtOAc in petroleum ether); $v_{\max }\left(\right.$ film) $3060,1769,1492,1448 \mathrm{~cm}^{-1} ; \delta_{\mathrm{H}}\left(300 \mathrm{MHz}, \mathrm{CDCl}_{3}\right) 7.38-7.25(5 \mathrm{H}, \mathrm{m}, \mathrm{Ph}), 4.66(1 \mathrm{H}$, $\mathrm{s},=\mathrm{CHH}), 4.64(1 \mathrm{H}, \mathrm{s},=\mathrm{CH} H), 2.93\left(1 \mathrm{H}, \mathrm{q}, J=6.6 \mathrm{~Hz}, \mathrm{CHCH}_{3} \mathrm{Ph}\right), 1.51(3 \mathrm{H}, \mathrm{d}, J=6.6 \mathrm{~Hz}$, $\left.\mathrm{CHCH}_{3} \mathrm{Ph}\right) ; \delta_{\mathrm{C}}\left(75 \mathrm{MHz}, \mathrm{CDCl}_{3}\right) 144.3(\mathrm{C}$, aryl), $137.4(=\mathrm{CN}), 128.8(\mathrm{CH}$, aryl $), 127.6(\mathrm{CH}$, aryl), $127.2\left(\mathrm{CH}\right.$, aryl), $83.5\left(=\mathrm{CH}_{2}\right), 68.8\left(\mathrm{CHCH}_{3} \mathrm{Ph}\right), 29.7\left(\mathrm{CD}_{2}\right), 23.9\left(\mathrm{CH}_{3}\right) ; \mathrm{MS}\left(\mathrm{CI}^{+}\right) \mathrm{m} / z$ $162\left(100 \%,[\mathrm{MH}]^{+}\right), 105\left(40 \%,\left[\mathrm{PhCHCH}_{3}\right]^{+}\right)$; $\mathrm{HRMS}\left(\mathrm{CI}^{+}\right)$calcd for $\mathrm{C}_{11} \mathrm{H}_{12} \mathrm{D}_{2} \mathrm{~N}[\mathrm{MH}]^{+}$ 162.1246, found 162.1244 . 


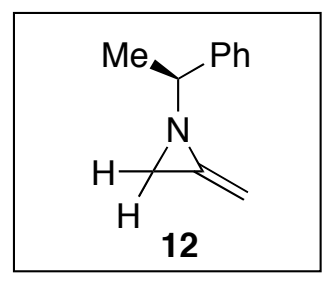

Preparation of 2-methylene-1-[(1S)-1-phenylethyl]aziridine 12 from (2-bromo-1,1-dideuteroprop-2-enyl)-[(S)-1-phenylethyl]-amine 10. A three-necked flask was fitted with a dry-ice condenser and a gas inlet. Iron(III) chloride $(2.0 \mathrm{mg}, 0.01 \mathrm{mmol}$ ) was added and the system was purged with $\mathrm{CaCl}_{2}$ dried ammonia. A dry-ice/acetone mixture was added to the condenser and ammonia $(25 \mathrm{~mL})$ condensed into the flask. Freshly cut sodium $(236 \mathrm{mg}$, $10.3 \mathrm{mmol}$ ) was added in small portions. The initially formed blue solution slowly faded to give a grey suspension of sodium amide. After cooling to $-78^{\circ} \mathrm{C}, \mathbf{1 0}(1.00 \mathrm{~g}, 4.13 \mathrm{mmol})[94 \% \mathrm{D}$ as judged by integration of the ${ }^{1} \mathrm{H}$ NMR signal at $2.70 \mathrm{ppm}$ ] in ether $(1 \mathrm{~mL})$ was added slowly to the mixture, which was then stirred for $1 \mathrm{~h}$. The mixture was diluted with diethyl ether $(5 \mathrm{~mL})$ and quenched by the dropwise addition of water $(2 \mathrm{~mL})$ (CAUTION). After the ammonia had evaporated, diethyl ether $(25 \mathrm{~mL})$ was added and the mixture was stirred for $2 \mathrm{~min}$. The organic phase was separated and washed with $0.1 \mathrm{M}$ acetic acid $(3 \times 50 \mathrm{~mL}), 10 \% \mathrm{NaOH}(2 \times 50 \mathrm{~mL})$ and brine $(50 \mathrm{~mL})$, then dried $\left(\mathrm{MgSO}_{4}\right)$. Removal of the solvent in vacuo and bulb-to-bulb distillation gave $12(601 \mathrm{mg}, 91 \%)$ as a clear colourless oil. Material judged to have $12 \% \mathrm{D}$ incorporation by integration of the signals at 2.11, 2.09, 2.01 and $2.00 \mathrm{ppm}$ in the ${ }^{1} \mathrm{H}$ NMR spectrum. $\delta_{\mathrm{H}}\left(300 \mathrm{MHz}, \mathrm{CDCl}_{3}\right) 7.38-7.25(5 \mathrm{H}, \mathrm{m}, \mathrm{Ph}) 4.66(1 \mathrm{H}, \mathrm{s},=\mathrm{CHH}), 4.64(1 \mathrm{H}, \mathrm{s}$, $=\mathrm{CHH}), 2.93\left(1 \mathrm{H}, \mathrm{q}, J=6.6 \mathrm{~Hz}, \mathrm{CHCH}_{3} \mathrm{Ph}\right), 2.15-2.07(0.94 \mathrm{H}, 2 \mathrm{x} \mathrm{s}$, aziridine $\mathrm{CHD}$ and $\mathrm{CHH})$, 2.04-1.98 $(0.82 \mathrm{H}, 2 \times \mathrm{x} \mathrm{s}$, aziridine $\mathrm{CDH}$ and $\mathrm{CHH}), 1.51\left(3 \mathrm{H}, \mathrm{d}, J=6.6 \mathrm{~Hz}, \mathrm{CHCH}_{3} \mathrm{Ph}\right)$. All other data consistent with those previously reported.

Sodium Amide Induced Exchange: Conversion of 11 to 12. A three-necked flask was fitted with a dry-ice condenser and a gas inlet. Iron(III) chloride $(2.0 \mathrm{mg}, 0.01 \mathrm{mmol})$ was added and the system purged with $\mathrm{CaCl}_{2}$ dried ammonia. A dry-ice/acetone mixture was added to the condenser and ammonia $(25 \mathrm{~mL})$ condensed into the flask. A small, freshly cut piece of sodium (18 $\mathrm{mg}, 0.78 \mathrm{mmol}$ ) was added to the flask and the initially formed blue solution slowly faded to give a grey suspension of sodium amide. $11(500 \mathrm{mg}, 3.10 \mathrm{mmol})$ [92\%D incorporation] in ether $(1 \mathrm{~mL})$ was added slowly to the mixture, which was then stirred for $1.5 \mathrm{~h}$. The mixture was diluted with diethyl ether $(5 \mathrm{~mL})$ and quenched by the dropwise addition of water $(2 \mathrm{~mL})$ (CAUTION). After the ammonia had evaporated, diethyl ether $(25 \mathrm{~mL})$ was added and the mixture was stirred for $2 \mathrm{~min}$. The organic phase was separated and washed with $10 \% \mathrm{NaOH}(2$ x $50 \mathrm{~mL}$ ) and brine $(50 \mathrm{~mL})$, dried over $\mathrm{MgSO}_{4}$ and the solvent removed in vacuo. Bulb-to-bulb distillation gave 12 (462 mg, 94\%) as a clear colourless oil. The material was judged to have $2.5 \% \mathrm{D}$ incorporation by integration of the signals at 2.11, 2.09, 2.01 and $2.00 \mathrm{ppm}$ in the ${ }^{1} \mathrm{H}$ NMR spectrum (average of two experiments). $\mathrm{R}_{\mathrm{f}} 0.52$ (10\% EtOAc in petrol); $v_{\max }$ (film) 3060, $1769,1492,1448 \mathrm{~cm}^{-1} ; \delta_{\mathrm{H}}\left(300 \mathrm{MHz}, \mathrm{CDCl}_{3}\right) 7.38-7.25(5 \mathrm{H}, \mathrm{m}, \mathrm{Ph}), 4.66(1 \mathrm{H}, \mathrm{s},=\mathrm{CHH}), 4.64$ 
$(1 \mathrm{H}, \mathrm{s},=\mathrm{CH} H), 2.93\left(1 \mathrm{H}, \mathrm{q}, J=6.6 \mathrm{~Hz}, \mathrm{CHCH}_{3} \mathrm{Ph}\right), 2.15-2.07(0.99 \mathrm{H}, 2 \mathrm{x} \mathrm{s}$, aziridine $\mathrm{CHD}$ and $\mathrm{CHH}), 2.04-1.98(0.96 \mathrm{H}, 2 \mathrm{x} \mathrm{s}$, aziridine $\mathrm{CDH}$ and $\mathrm{CH} H), 1.51\left(3 \mathrm{H}, \mathrm{d}, J=6.6 \mathrm{~Hz}, \mathrm{CHCH}_{3} \mathrm{Ph}\right)$; $\delta_{\mathrm{C}}\left(75 \mathrm{MHz}, \mathrm{CDCl}_{3}\right) 144.3(\mathrm{C}$, aryl $), 137.4(=\mathrm{CN}), 128.8(\mathrm{CH}$, aryl $), 127.6(\mathrm{CH}$, aryl $), 127.2$ $(\mathrm{CH}$, aryl $), 83.5\left(=\mathrm{CH}_{2}\right), 68.8\left(\mathrm{CHCH}_{3} \mathrm{Ph}\right), 29.8\left(\mathrm{NCH}_{2}\right), 23.9\left(\mathrm{CH}_{3}\right) ; \mathrm{MS}\left(\mathrm{EI}^{+}\right) \mathrm{m} / z 159(10 \%$, $\left.\mathrm{M}^{+}\right), 105\left(100 \%,\left[\mathrm{PhCHCH}_{3}\right]^{+}\right)$; HRMS $\left(\mathrm{ES}^{+}\right)$calcd for $\mathrm{C}_{11} \mathrm{H}_{14} \mathrm{~N}[\mathrm{MH}]^{+}$160.1121, found 160.1122 . 